\title{
Modulare Stahllamellenhallen von Hugo Junkers
}

\section{Wissenschaftliche Grundlagen für eine denkmalgerechte Instandsetzung}

Der für seine bahnbrechenden Erfindungen im Flugzeug- und Anlagenbau bekannte Ingenieur HUGO JUNKERS (1859-1935) entwickelte in den 1920er-Jahren innovative Hallenkonstruktionen aus modularen Stahlblechlamellen, die weltweit vermarktet und gebaut wurden. Sie stehen in engem Kontext zur ebenfalls patentierten Holzbauweise FRIEDRICH ZOLLINGERS (1880-1945). Die Bauweise gilt als Meilenstein in der Entwicklung des modularen Leichtbaus und bedeutendes Zeugnis der Moderne. Bedauerlicherweise sind nur noch wenige dieser Bauwerke erhalten.

Auf dem Gelände des ehemaligen Militärflugplatzes Oberschleißheim stehen derzeit noch zwei dreischiffige, tonnenförmige Hallen von 1934. Die Hallen stehen unter Denkmalschutz, zeigen jedoch einen bedenklichen baulichen Zustand. Sie gaben den Anlass für ein Forschungsprojekt, das von 20142016 am Lehrstuhl für Tragwerksplanung der Technischen Universität München unter Förderung der Meitinger Stiftung bearbeitet wurde.

Die wesentlichen Ergebnisse der Untersuchungen werden im Rahmen des Artikels zusammengefasst: Exemplarisch wurde ein Hallenschiff in Oberschleißheim mit einem 3-D-Laserscan vermessen und die Punktwolke in ein statisches Rechenmodell überführt. Parametrische Analysen brachten neue Erkenntnisse zur Geometrie und Fügung des Konstruktionsprinzips. Studien zum Tragverhalten zeigen verschiedene Schwachpunkte der Struktur auf, die für eine Instandsetzung unbedingt berücksichtigt werden müssen.

Keywords Hugo Junkers; Lamellenhalle; Stahlblech; modular; Tonnenschale; Tragverhalten; Instandsetzung

\section{$1 \quad$ Historischer Abriss}

Hugo JunKers wird 1859 als drittes von sieben Kindern im nordrheinwestfälischen Rheydt bei Düsseldorf geboren. Von 1878-1883 studiert er in Berlin und Aachen Maschinenbau. Nach dem Studium arbeitet JunKERs zunächst als Angestellter in verschiedenen Unternehmen und entwickelt 1892 den ersten Zweitakt-GegenkolbenGasmotor sowie das Kalorimeter zur Bestimmung des Heizwerts. Das Patent für den ersten Gasbadeofen als Vorgänger des Durchlauferhitzers erhält er bereits mit seiner eigenen Firma Junkers \& Co im Jahre 1894. Es folgen diverse Firmenaus- und -neugründungen, vorwie-

*) Corresponding author: joram.tutsch@tum.de

Submitted for review: 09 August 2016

Revised: 11 January 2017

Accepted for publication: 26 January 2017
Modular steel lamella structures by Hugo Junkers - scientific bases for an appropriate repair

The German engineer HUGO JUNKERS (1859-1935) is well-known mostly for his innovations in plant engineering and aircraft design. In the mid-1920s, he developed an innovative roof construction that was comprised of modular steel lamellas, and is regarded a milestone in the development of lightweight structures. The technical construction principle is closely related to ZOLLINGER's (1880-1945) timber lamella roof design. Unfortunately, most of the buildings do not exist anymore or are in bad condition.

Two three-section, barrel-vaulted hangars from 1934 still exist on the site of the former military airfield in Oberschleißheim. They are classed as listed buildings but their structural condition is very alarming. They gave the occasion of a research project, promoted by the Meitinger Foundation, which has been edited from 2014-2016 at the Chair of Structural Design at the Technical University of Munich.

This article summarizes the main results of the investigations: The point cloud of a 3-D-laser-scanning was converted into a static calculation model. Parametric analyzes brought new insights into the geometry and fitting of the construction. Studies on the load bearing behavior show a number of weaknesses of the structure, which necessarily have to be considered in a maintenance.

Keywords Hugo Junkers; lamella roof; steel; modular; barrel vault; load bearing; maintenance

gend am Standort Dessau, ehe er 1897 die ordentliche Professur für Thermodynamik an der Technischen Hochschule Aachen antritt. Über seinen Kollegen Hans REIssNER wendet sich JunKERs zusehends dem Flugzeugbau zu und entwickelt 1910 den „dicken Flügel“ - die erste Tragfläche vollständig aus Metall. Fünf Jahre später gelingt ihm die bahnbrechende Erfindung der ersten Ganzmetallflugzeuge, die einen nicht für möglich gehaltenen Quantensprung in der Luftfahrt darstellt.

Er gründet 1919 die erste zivile Fluglinie zwischen Dessau und Weimar und zwei Jahre später die Junkers-Luftverkehrs AG, die schließlich in der Deutschen Lufthansa aufgeht.

Die deutlich vergrößerten Spannweiten der neuen Flugzeuggeneration erfordern Hangar-Konstruktionen, die zum damaligen Zeitpunkt so noch nicht existieren. Mit 


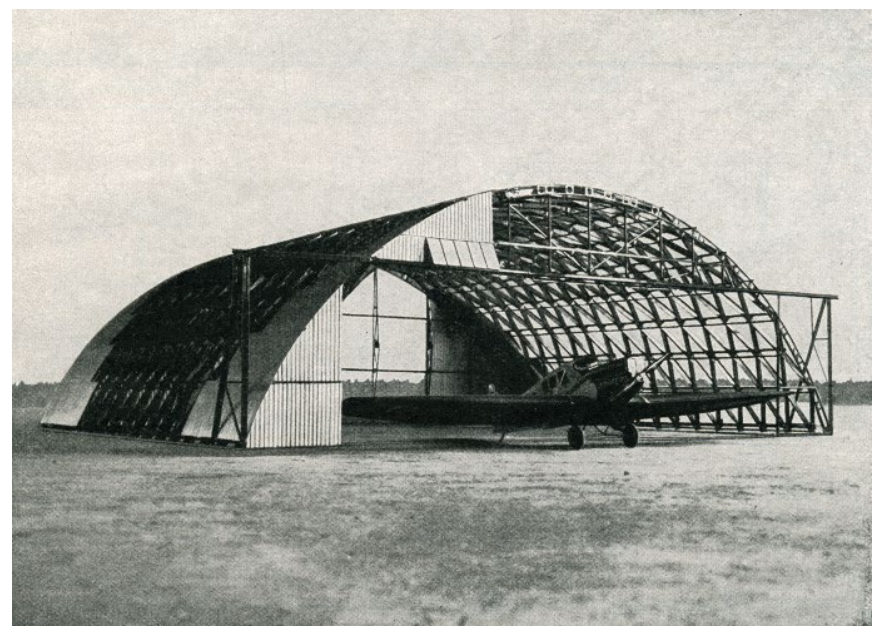

Bild 1 Probeaufbau eines Hangars auf dem Junkers-Werksgelände in Dessau

Test installation of a hangar on the Junkers' factory site

seiner firmeneigenen Bauabteilung entwirft JunKERs Tonnenhallen aus Stahlblechen (Bild 1). Dabei greift er auf sein fortschrittliches Wissen im Blechbau zurück, das er im Zuge der Flugzeugentwicklung gewonnen hat. Anfänglich nur für interne Zwecke verwendet, erkennt er Ende der 1920er-Jahre das Potenzial der Konstruktionsweise und entwickelt sie $\mathrm{zu}$ einem Massenprodukt (Bild 2), das weltweit vermarktet und gebaut wird.

Auf dem Zenit seines unternehmerischen Erfolgs stellt sich JuNKERS öffentlich gegen das nationalsozialistische
Regime und dessen Rädelsführer. Innerhalb seines gigantischen Firmenkonsortiums wird er binnen weniger Monate Opfer verschiedener Intrigen, verliert Mehrheitsanteile an Unternehmensbereichen und zieht schließlich 1932 mit den ihm verbliebenen Abteilungen nach München-Allach um. Doch auch dieser vermeintliche Frieden währt nicht lange - JunKers wird weiter (firmen-)politisch isoliert, 1933 enteignet und unter Hausarrest gestellt und stirbt schließlich 1935 an seinem 76. Geburtstag in seinem Haus in München-Gauting [1, 2].

\section{Konstruktionsweise}

In den folgenden Abschnitten werden Erkenntnisse zum vertieften Verständnis der Konstruktion der Lamellenhallen hinsichtlich ihrer Geometrie, der Bauteilfügung, der Werkstoffwahl und der Bauaufnahme sowie zu typischen Schadensbildern und dem Tragverhalten dargelegt. Dabei handelt es sich um verschiedene Aspekte, die im Zuge der Untersuchungen der Hallen in Oberschleißheim gemacht wurden, ohne dabei Anspruch auf Vollständigkeit zu erheben. Die innovations- und konstruktionshistorische Entwicklung der Bauweise wurde bereits in einem umfangreichen Diskussionsbeitrag aufgearbeitet [3].

\subsection{Form und Fügung der Lamellenstruktur}

Die Stahllamellenhallen beruhen auf der Geometrie einer einfach gekrümmten Tonne. Dies ermöglicht die modula-
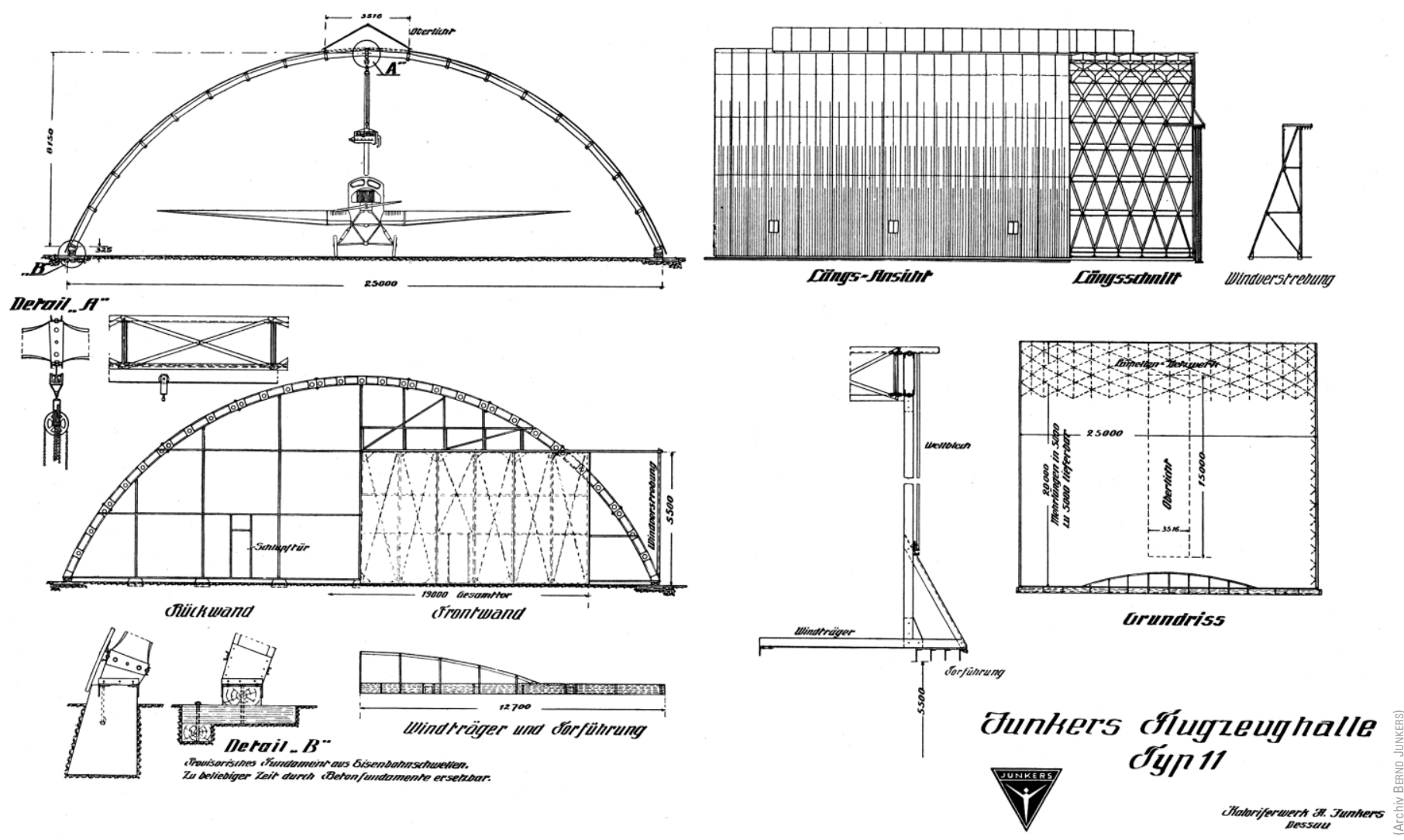

Bild 2 Konstruktionsschema einer schlüsselfertigen Halle „Typ 11“ Construction scheme of hangar "Typ 11" 


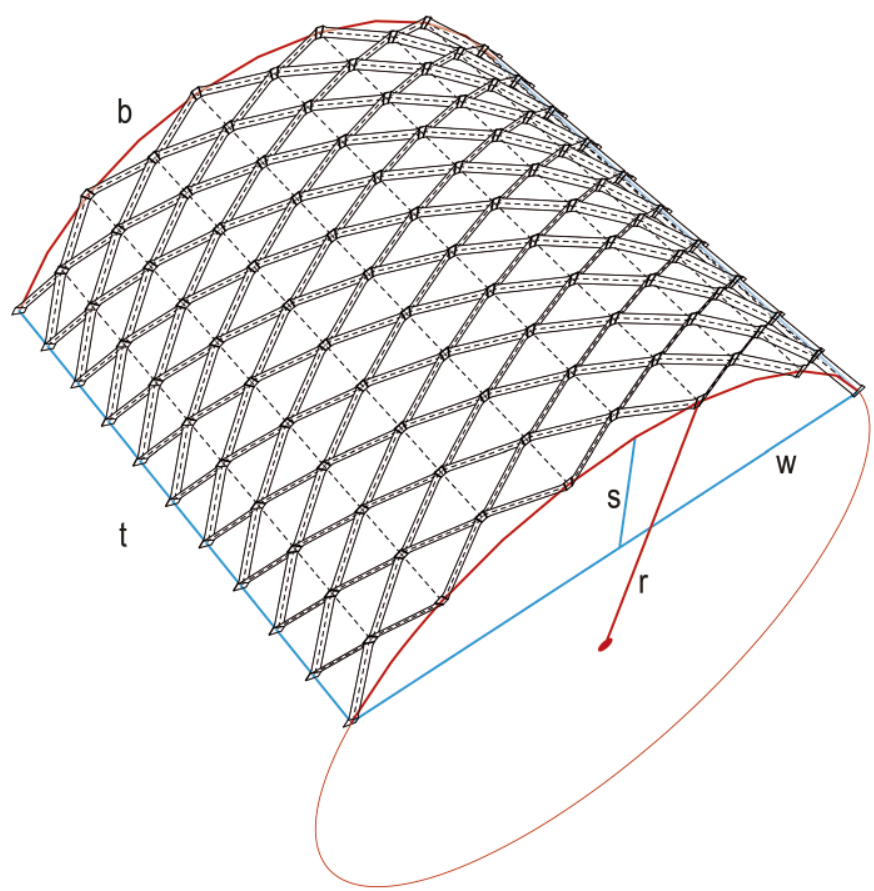

Bild 3 Axonometrie der Junkershalle Junkers hall barrel-vaulted axonometry

re Konstruktionsweise mit identischen Lamellen und Anschlusswinkeln.

Die Form des Zylinder-Ausschnitts ist abhängig von der Spannweite $w$ und dem Stich $s$. Sie kann über den Radius $r$, die Bogenlänge $b$ und die Tiefe $t$ definiert werden (Bilder 3, 4). Die Länge $l$ und der Öffnungswinkel $\alpha$ der Lamellen geben das Raster in $x$ - und $y$-Richtung vor. Bogenlänge und Tiefe sind so gewählt, dass sie ein Vielfaches dieses Rasters ergeben.

Die Bogengeometrie ist nicht perfekt rund. Sie wird durch einen regelmäßigen Polygonzug angenähert. Tatsächlich liegen nur die Knotenpunkte auf der Zylindergeometrie. Das diagonale Raster verbindet die Knotenpunkte auf ebener Strecke und gleicht sich mit einem konstanten Rotationswinkel $\beta$ der Bogenform an.

Die Lamellen sind senkrecht zur Tonne ausgerichtet. Dies führt dazu, dass die $z$-Achsen der Lamellenprofile von der globalen $z$-Achse abweichen. Je stärker die Neigung der Tonne ist, umso höher ist diese Abweichung. Der Effekt lässt sich in der Aufsicht (Bild 4) deutlich ablesen. Zum Auflager hin werden die seitlichen Flanken der Lamellen zunehmend sichtbar.

Die Betrachtung einer einzelnen Lamelle bringt Aufschluss über ihre Ausrichtung und die Geometrie der Knotenpunkte (Bilder 5, 6). Eine Lamelle verbindet zwei diagonal gegenüberliegende Punkte $A$ und $B$. Die Knotenachsen $N_{\mathrm{A}}$ und $N_{\mathrm{B}}$ stehen senkrecht zur Tonne. Das heißt, sie verbinden das Zentrum der parallelen Kreisbögen $\left(B_{\mathrm{A}}\right.$ bzw. $\left.B_{\mathrm{B}}\right)$ mit den Punkten $(A$ bzw. $B)$ auf der Tonnenfläche. Sie liegen also in zwei unterschiedlichen Ebenen und stehen windschief zueinander. Eine gerade
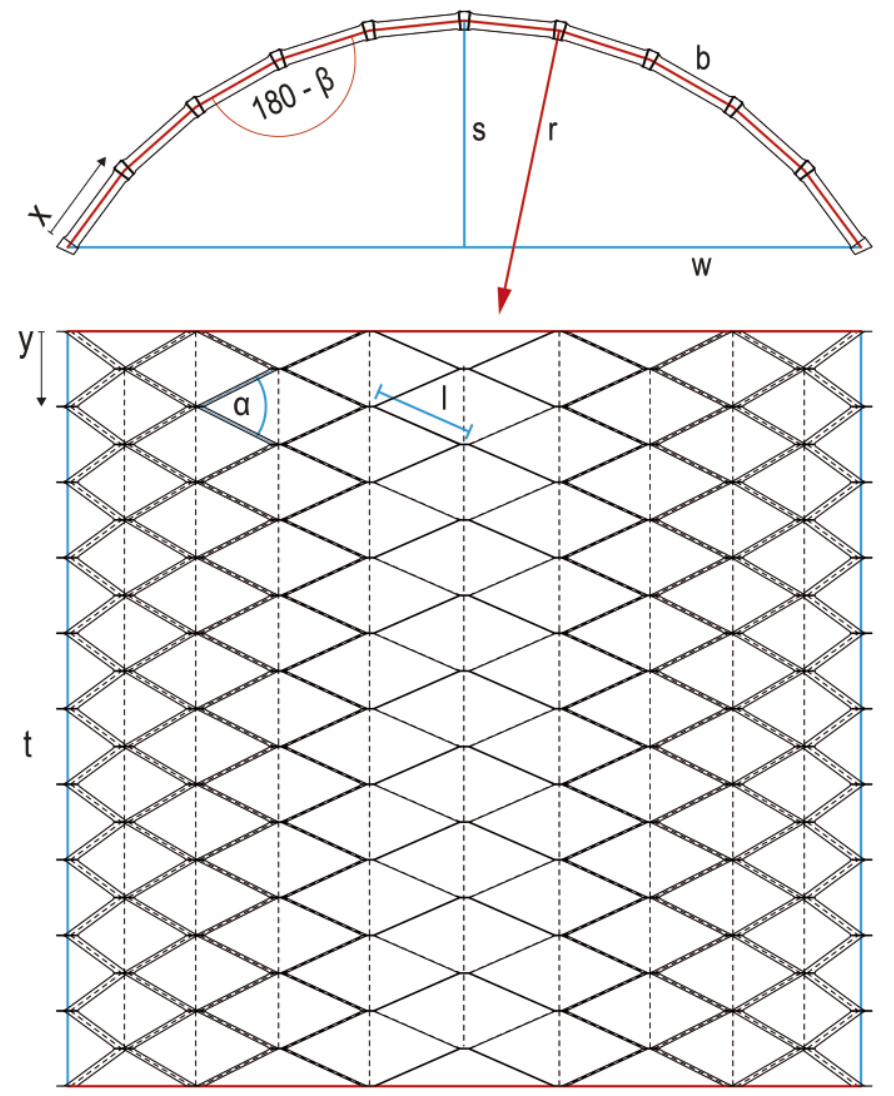

Bild 4 Tonnengeometrie der Junkershallen in An- und Aufsicht Junkers hall barrel-vaulted geometry in front and side view

Lamelle müsste tordiert/verwunden werden, um die Achsen $N_{\mathrm{A}}$ und $N_{\mathrm{B}} \mathrm{zu}$ verbinden.

JUNKERs löst dieses konstruktive Problem mit einem einfachen Knick. Die $z$-Achse des Lamellenprofils ist normal zur Tonne, parallel zur Achse $N_{\mathrm{L}}$ ausgerichtet. Die Lamelle ist nicht verwunden und verläuft gerade zwischen den Ebenen von $B_{\mathrm{A}}$ und $B_{\mathrm{B}}$. Dort knickt sie um den Öffnungswinkel $\alpha \mathrm{ab}$. Dabei verläuft die Knickachse $K_{\mathrm{A}}$ parallel zur Achse $N_{\mathrm{L}}$. Der abgeknickte Teil der Lamelle liegt nun in der Ebene von $B_{\mathrm{A}}$ bzw. $B_{\mathrm{B}}$. Auf diese Weise treffen am Knotenpunkt vier Lamellen ohne Verwindung parallel aufeinander.

Der Rotationswinkel $\beta$ wird im Knoten aufgenommen. Er ist zwischen zwei gegenüberliegenden Knickachsen $K_{\mathrm{A}}$ und $K_{\mathrm{A}}$ ' ablesbar und wird im Zuschnitt und den Schraubenlöchern der Lamelle berücksichtigt. Die Knotenachse $N_{\mathrm{A}}$ bildet die Winkelhalbierende. Die Lamellen-Backen sind auf Ober- und Unterseite nicht bündig. Sie kreuzen sich ebenfalls im Winkel $\beta$.

\subsection{Verbindungsdetail}

Bild 7 zeigt alle relevanten Bauteile und deren Verbindungen. In einem Knoten treffen sich jeweils vier Lamellen (a), dort werden sie mittels Backenblechen (b) über drei bis sechs Schrauben verbunden. Diese - an der Oberund Unterseite aufgekanteten - Bleche bieten wiederum 


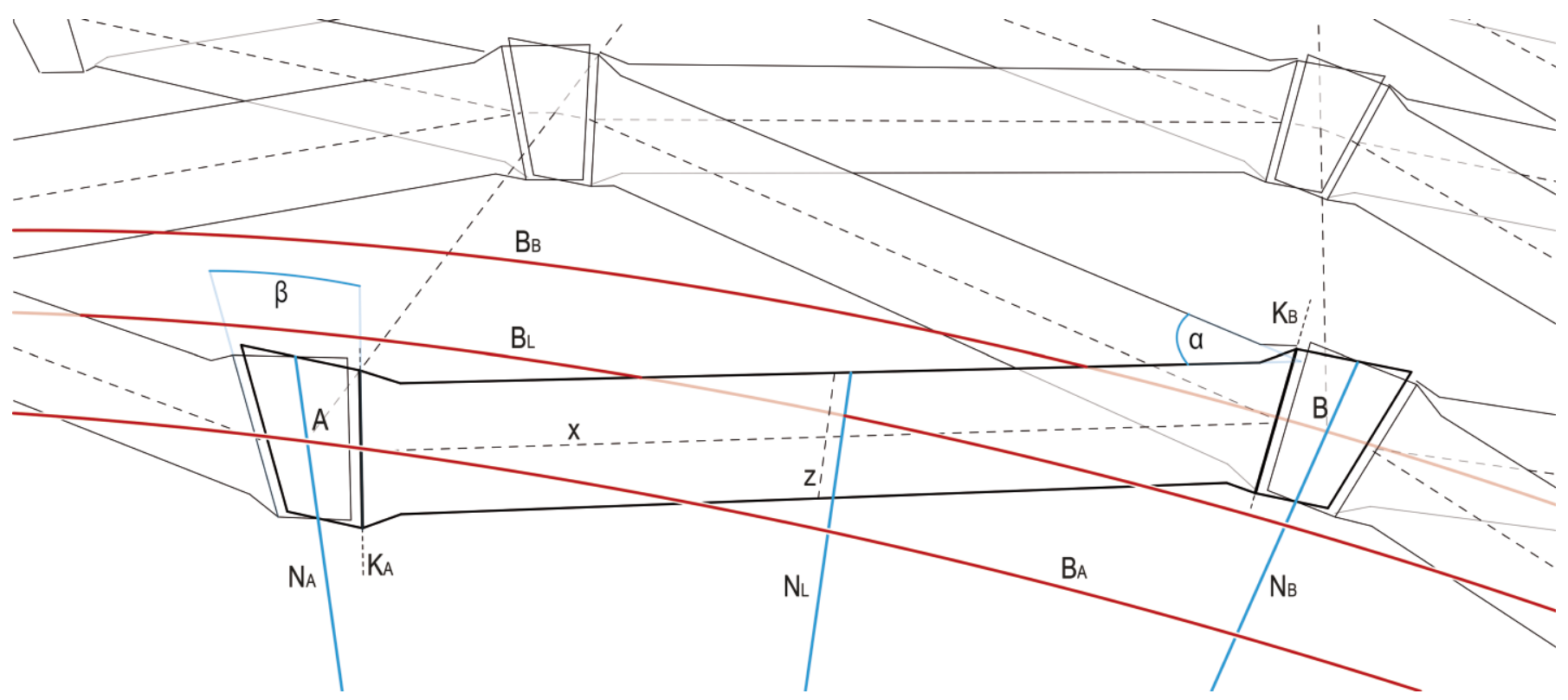

Bild 5 Fügungsgeometrie der Lamellen Fitting of lamellas

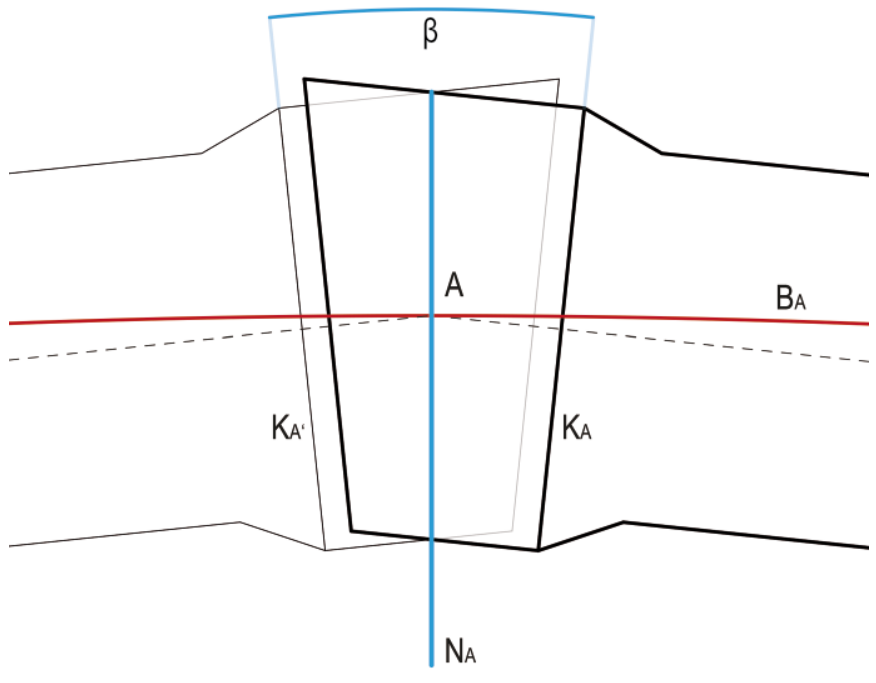

Bild 6 Überlappung der abgeknickten Bleche am Knotenpunkt Overlapping of kinked sheets at the junction

die Möglichkeit, die Ober-und Unterpfette (c, d) anzuschließen.

\section{$2.3 \quad$ Material}

Sowohl im Flugzeug- als auch im Hochbau setzte JuNKERS hauptsächlich kaltverformte Profile ein, also dünne Stahlbleche, die in die gewünschte Form gepresst wurden [4]. Alle Bauteile des Lamellendachs (Lamellen, Pfetten, Backenbleche) wurden auf diese Weise hergestellt (Bild 8). Allerdings muss hier zwischen Fein- und Grobblech $(<3,00 \mathrm{~mm}$ bzw. $>3,00 \mathrm{~mm})$ unterschieden werden (Regelung bis 1981: Mittelblech 3,00 mm $<t<4,75 \mathrm{~mm}$ ). Für Auflager- und Anschlussbauten, sofern diese ebenfalls aus Stahl errichtet wurden, kamen vorwiegend Walzprofile zum Einsatz. Da das Rohmaterial i. d. R. Meterware war, also keinerlei Einprägungen oder sonstige Kenn-

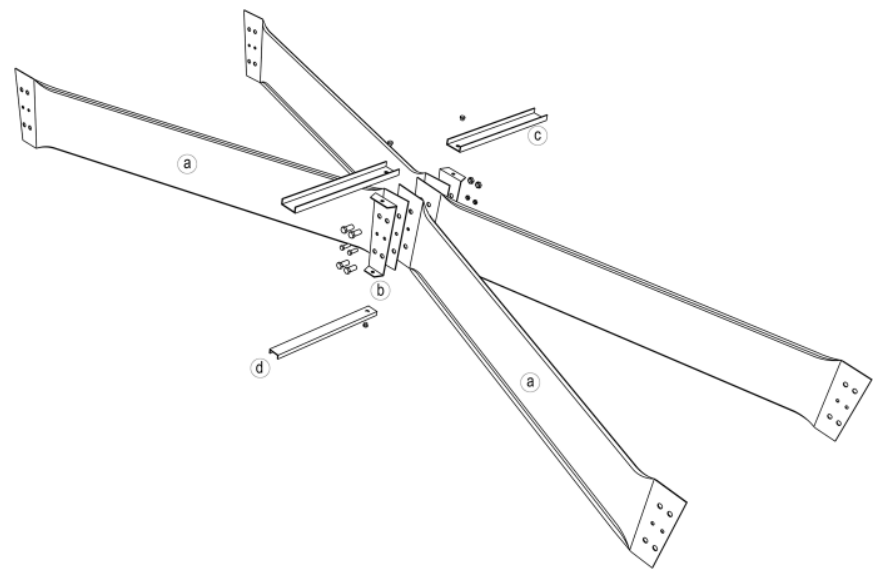

Bild 7 Explosionszeichnung des Verbindungsdetails: a) Lamelle, b) Backenblech, c) Oberpfette, d) Unterpfette

Exploded drawing of junction: a) lamella, b) cover plates, c) top purlin, d) bottom purlin

zeichnung enthält, ist es heute quasi unmöglich, ohne eine entsprechende Werkstoffanalyse dessen genaue Qualität zu bestimmen. Auch Archivrecherchen sowie Anfragen bei verschiedenen Folgeunternehmen der Junkerswerke blieben erfolglos.

Am Beispiel einer Unterpfette $(t=2,00 \mathrm{~mm})$ der Hallen in Oberschleißheim - eine Lamelle kann ohne zusätzliche Sicherungsmaßnahmen nicht ausgebaut werden - wurde eine umfassende Werkstoffanalyse in Form von Zugversuchen, Härtemessungen, Gefüge- und Elementaranalyse durchgeführt:

„Die Untersuchungsergebnisse legen nahe, dass es sich bei dem [...] verwendeten Blech um ein Feinblech nach DIN 1623 (Mai 1932) mit der Bezeichnung St 37.23 handelt, was für Feinbleche mit vorgeschriebener Festigkeit, z. B. für Stanzteile, empfohlen wurde. Der Stahl entspricht damit hinsichtlich der Festigkeit einem [heuti- 

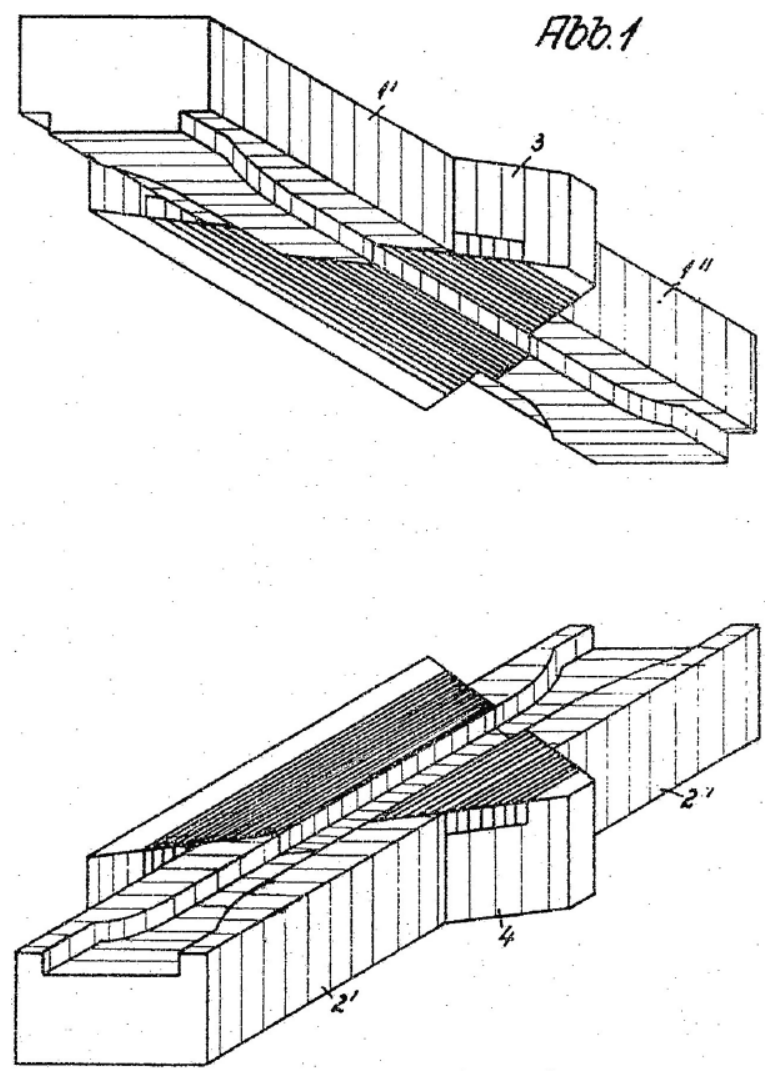

Bild 8 Patentzeichnungen für das „Preßwerkzeug zur Herstellung von Netzwerkstäben aus Blech“" [5]

Patent drawing [5]

gen] S235JR, Werkstoffnummer 1.0038, nach DIN EN 10025-1/-2 [...], der heutzutage aber immer beruhigt vergossen wird und einen höheren Kohlenstoffgehalt (ca. 0,17 Gew.-0) besitzt“ [6].

Prinzipiell sollte auf Grundlage der Werkstoffanalyse eines Bauteils der Hallen jedoch nicht pauschal von derselben Materialqualität für alle weiteren Bauteile ausgegangenen werden. Die Lamellen in Oberschleißheim haben beispielsweise eine Blechstärke von $t=5,00 \mathrm{~mm}$, sind damit also im Gegensatz zu den Feinblechen der Unterpfetten ein Grobblech und können theoretisch signifikant unterschiedliche Eigenschaften besitzen.

3

\section{Typische Schäden der Hallen}

In Oberschleißheim konnten folgende Schadensbilder dokumentiert werden (Bilder 9, 10):

- Fehlende oder stark verformte Unterpfetten

- Verdrehung der Knoten um die lokale Knoten-Achse $\left(N_{\mathrm{A}}\right.$ bzw. $\left.N_{\mathrm{B}}\right)$

- Ausgeknickte Lamellen um die lokale (schwache) z-Achse

- Flächige Korrosion mit variierender Intensität

- Einschusslöcher in allen Bauteilen

Mit Ausnahme der Einschusslöcher können diese Schäden als typisch für die Bauweise betrachtet werden. Jüngere Untersuchungen von Hallen in Dessau und England

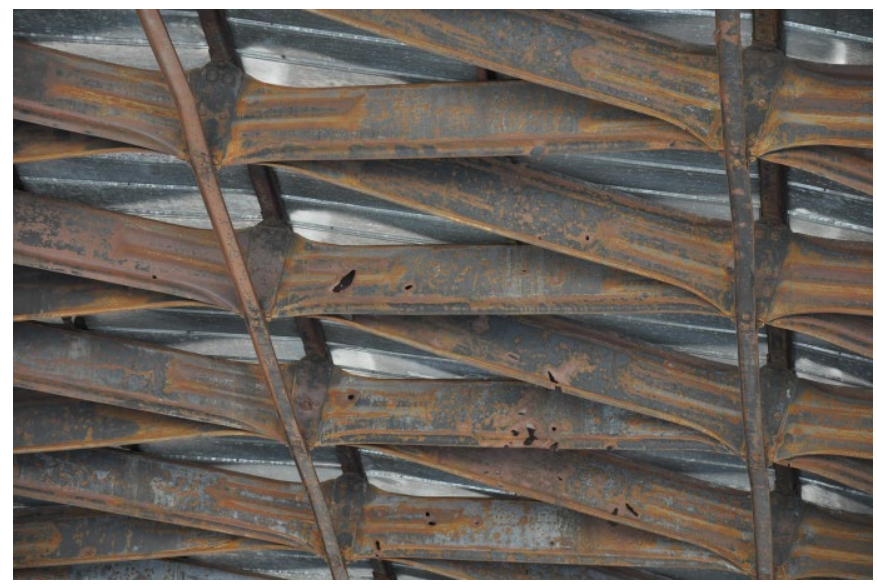

Bild 9 Typische Schäden am Hangardach in Oberschleißheim: Korrosion und Einschusslöcher

Typical damages of Oberschleißheim hangar roof: corrosion and bullet holes

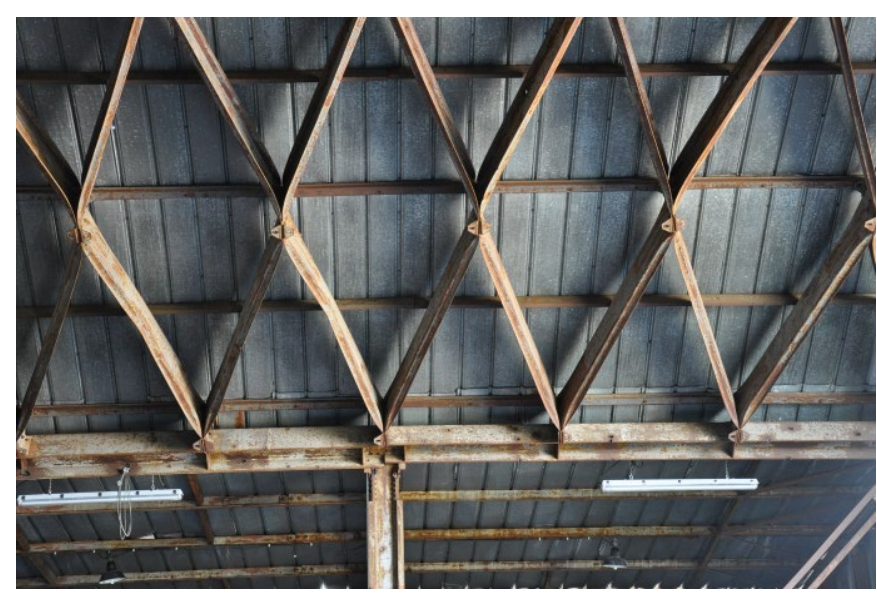

Bild 10 Typische Schäden am Hangardach in Oberschleißheim: fehlende Unterpfetten, geknickte Lamellen, verdrehte Knoten Typical damages of Oberschleißheim hangar roof: missing bottom purlins, buckled lamellas, twisted junctions

weisen analoge Beobachtungen auf [7, 8]. Allerdings sind die mechanischen Verformungen phänomenologisch von der Korrosion zu unterscheiden, deren Hauptursache in undichten Gebäudehüllen und mangelhafter Instandhaltung (Anstrich) zu sehen ist. Schon bauzeitlich galt insbesondere die allseits mögliche Luft-Umströmung der statischen Bauteile als eine der wichtigsten korrosionsreduzierenden Eigenschaften [9], was sich auch weitestgehend bestätigt hat.

Der Grund für die verschiedenen Deformationen der Bauteile kann aus der Systematik der Konstruktionsweise abgeleitet werden und wird in nachfolgendem Abschnitt detailliert erörtert.

\section{Tragverhalten}

\subsection{Globalstatisches Modell}

Eine Tonne - egal ob als kontinuierliche Fläche oder aufgelöste Gitterstruktur - kann an ihren Längsseiten, 
also entlang des Kämpfers, oder zusätzlich an ihren Stirnseiten gelagert werden. In der ersten Variante entspricht das Tragverhalten für in Längsrichtung gleichverteilte Lasten (Eigengewicht, Schnee) dem von aneinandergereihten Zwei-Gelenk-Bögen, da eine Schubtragwirkung (= Schalentragwirkung) nicht erforderlich ist. Bei der zweiten Lagerungsmöglichkeit und insbesondere bei ungleichen Lasten (z. B. Wind, lokale Schneeanhäufung) muss die Tonne auch Schubkräfte übertragen [10]. Beide Lagerungstypen fanden bei den Junkershallen Anwendung, wobei eine giebelseitige Abstützung eher die Ausnahme darstellt. Bei der überschlägigen Analyse des Tragverhaltens kann daher eine zweidimensionale Betrachtung als Zwei-Gelenk-Bogen als ausreichend angesehen werden (Bild 11).

Üblicherweise wurde das Tonnendach mithilfe verschiedener Konstruktionen vom Baugrund abgehoben, seltener setzte der Kämpfer direkt auf einem Fundament auf. Ein solches Übergangsbauwerk wirkt als mehr oder weniger elastisches Auflager für die Tonne. Idealerweise sollte jeder Randknoten möglichst gleich gelagert werden, da unterschiedliche Lagersteifigkeiten den Kraftfluss in der Tonne nennenswert beeinflussen. Gerade bei einer Bewertung der Resttragfähigkeit sollte also der Lagersituation eine besondere Aufmerksamkeit zukommen.

\subsection{Systematik des Stabnetzes}

Wird das System gedanklich eben (2-D) abgewickelt, kann seine statische Wirkungsweise anschaulich erklärt werden (Bild 12). Ein Dreieck aus zwei Lamellen, die am Endpunkt verbunden sind und am Anfangspunkt je ein festes Auflager besitzen, bildet eine statisch bestimmte Grundstruktur. Dieses Dreieck wiederholt sich in der ersten Reihe beliebig oft, je nach Tiefe der Tonne. Nun wird eine zweite Reihe an die Endpunkte der ersten angeschlossen: die Endpunkte aus Reihe 1 stellen je ein festes Auflager für die Lamellen der Reihe 2 dar. Dieses Vorgehen kann so oft wiederholt werden, wie Dreiecke in Reihe 1 vorhanden sind und schlussendlich ein „großes Dreieck“ geformt ist (ebenes Fachwerkbildungsgesetz). Soll nun ein rechteckiger Grundriss entstehen, ist das Anbringen einer weiteren Lamelle nur möglich, wenn ein Bauteil parallel zur Kämpferlinie an den Endpunkt der neuen Lamelle hinzugefügt wird. Diese Funktion übernehmen die Pfetten (Bild 12).

Um die Stabilität auch für die räumliche (3-D) Tonne zu gewährleisten, müssen die Verbindungen zwischen den Lamellen um die Achse parallel zur Kämpferlinie biegesteif ausgeführt werden. Konstruktiv wird dies durch ein oder mehrere Schraubenpaare am Verbindungspunkt realisiert. Für den Lamellenanschluss in den Bereichen außerhalb der „großen Dreiecke“ übernimmt diese Funktion ein Kräftepaar in Ober- und Unterpfette.

\subsection{Lokale Probleme}

Für gleichförmige Lasten könnten die auftretenden Schnittgrößen problemlos über das für den Herstellungsprozess und Bauablauf optimierte Verbindungsdetail übertragen werden (Bild 11). Verteilen sich die Kraftgrößen auf die Lamellen ungleich, kommt es aufgrund des
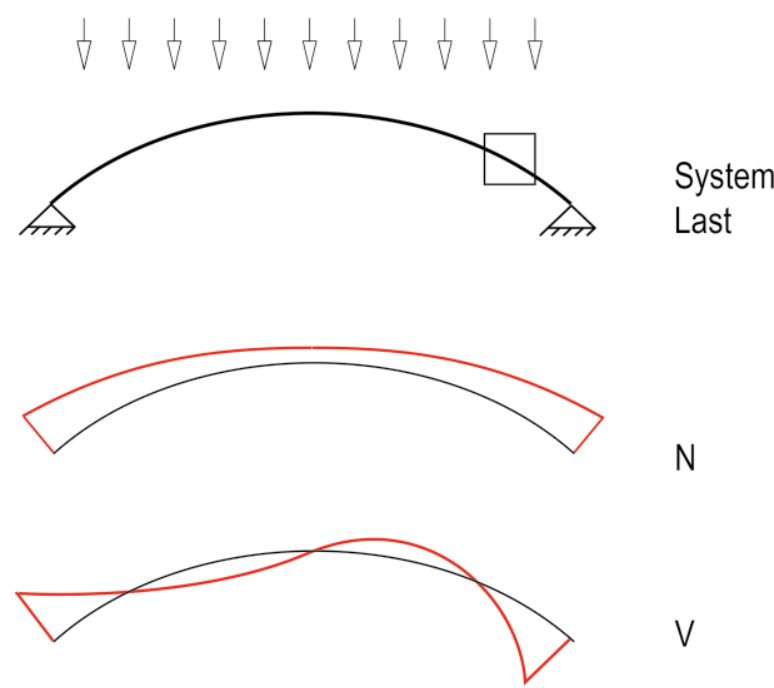

$\mathrm{N}$

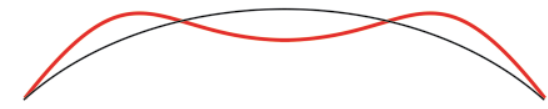

System

$\mathrm{M}$
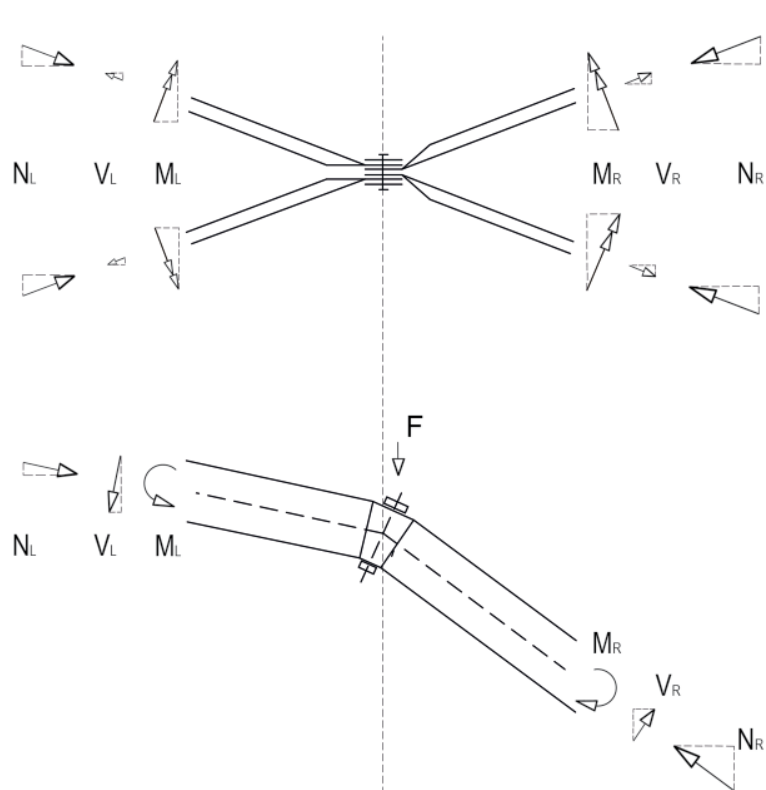

Bild 11 Zwei-Gelenk-Bogen und prinzipieller Schnittgrößenverlauf für gleichverteilte Lasten (links), Knotengleichgewicht in Auf- und Ansicht (rechts) Two-hinged arch with internal forces for uniform loads (left), equilibrium of forces at junction - top view and elevation (right) 

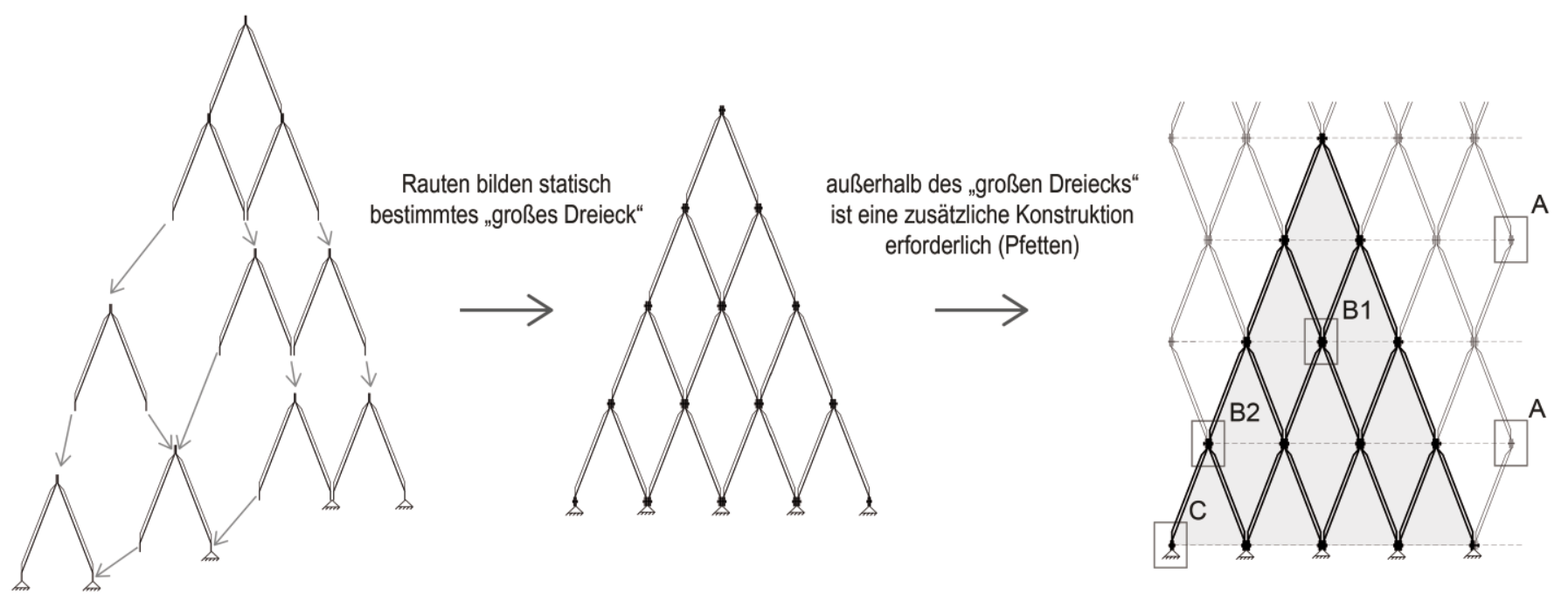

A: Randbereich
Kräftepaar in Pfetten
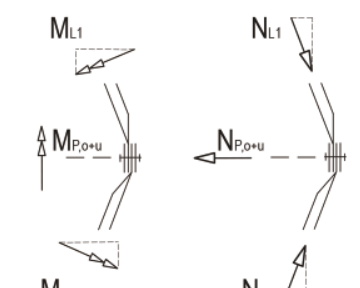

Mเ2

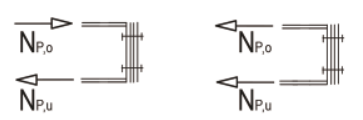

B1: Regelknoten symmetr. Last

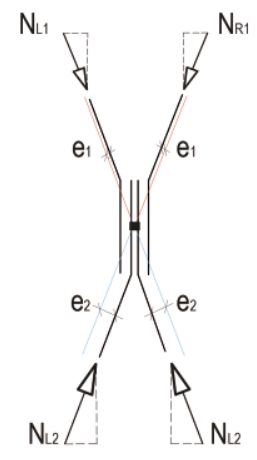

B1: Regelknoten asymmetr. Last

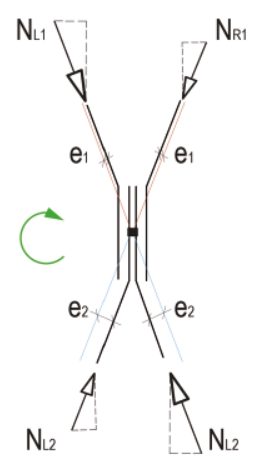

B2: Randknoten Rotationsgefahr

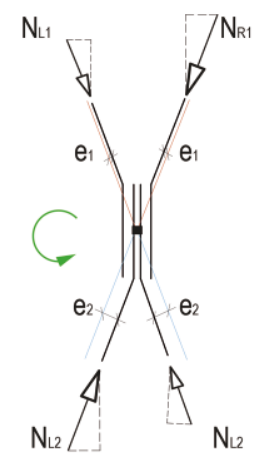

C: Randlager Rotationsgefahr

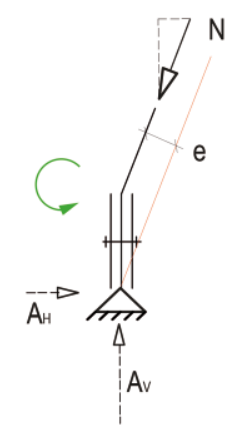

Bild 12 Entwicklung des Systems aus statisch bestimmten Dreiecken (oben), Kräftegleichgewicht in Abhängigkeit von Last und Knotenposition (unten) Structural development out of statically determinate triangles (above), equilibrium of forces depending on load and node position (below)

exzentrischen Anschlusses zu Biegemomenten um die schwache Achse der Profile, welche nur bedingt an der Koppelstelle weitergeleitet werden können (Bild 12). Ursächlich für diese Art der Lastverteilung ist eine Schubbeanspruchung der Tonne aus ungleicher Belastung und/ oder ungleichen Auflagerbedingungen. Übersteigt diese Biegung den Bauteilwiderstand, hat dies vor allem im Auflager- und Randbereich die oben beschriebene Knotenverdrehung zur Folge. Dieses Phänomen kann eine Art Kettenreaktion auslösen, da die Lamellen, die sich der Kraft „entzogen haben“, nicht mehr zum globalen Lastabtrag beitragen. Die Druckkraft und damit die Knickgefahr der umliegenden Lamellen steigt sprunghaft an.

Die Ingenieure um JunKers gingen davon aus, dass die filigranen Pfetten nur auf Zug belastet werden [11, 12]. Durch den oben beschriebenen Effekt aus Schalenschub aufgrund des geraden Randabschlusses (Bild 12) und aus lokaler Einleitung von Windlasten auf die Giebelseiten der Hallen kann in diesen Bauteilen auch eine Druckkraft entstehen. Ihr Knickwiderstand ist relativ gering, was - wie in Oberschleißheim der Fall - zu frühem Stabilitätsversagen führen kann.
Die zum Kämpfer zunehmende räumliche Kippung der Lamelle (Abschn. 2.1) wirkt sich für Gravitationslasten (Eigengewicht, Schnee) negativ aus, da zwangsläufig Biegung um die schwache Achse entsteht. Für Windlasten gilt dieses Phänomen nicht, da der Winddruck/-sog senkrecht zur Tonne angreift.

\section{$5 \quad$ Methodik der Instandsetzung}

\subsection{Sofortsicherungsmaßnahmen}

Wenn für eine Halle akute Einsturzgefährdung attestiert wird, besteht unmittelbarer Handlungsbedarf. Eine geeignete Notsicherung muss kurzfristig hergestellt werden. Da solche temporären Maßnahmen häufig über mehrere Jahre die Standsicherheit der Konstruktion gewährleisten müssen, ist trotz der gebotenen Eile eine sorgfältige Planung angeraten. Mitunter können unsachgemäße Abstützungen oder Verstärkungen sogar negative Auswirkungen auf das Tragverhalten haben. So sind insbesondere einzelne, punktuelle Unterstützungen der Verbindungsknoten zu vermeiden, da dies ggf. zu einer erhöhten Biegebelastung einzelner Bauteile führen kann. 


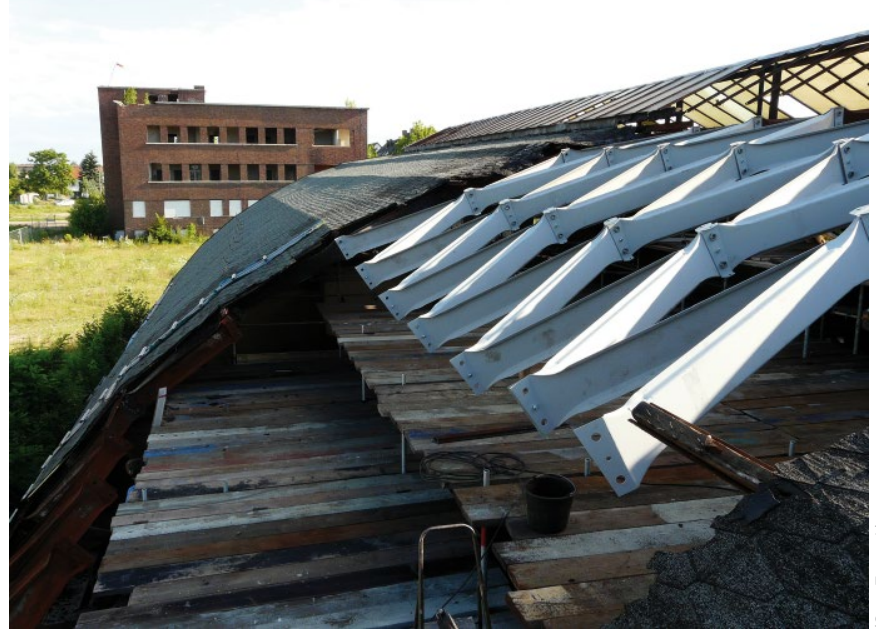

Bild 13 Austausch von Bauteilen einer Halle auf dem ehemaligen JunkersWerksgelände in Dessau

Exchange of components of a hall on the former Junkers' factory site in Dessau

\subsection{Behebung prinzipieller Mängel}

Die in Abschn. 4.3 beschriebenen systeminhärenten Schwachstellen der Struktur sollten als mögliche Schadensursache überprüft werden. Gerade der Anschluss bzw. Nichtanschluss an die Giebelwand sollte funktionsfähig konstruiert sein. Eine Kopplung von Tonne und Wand sollte nur dann erfolgen, wenn beide Bauteile entsprechend ausgebildet sind resp. für diesen Zweck verstärkt werden können. Zudem sollten alle freien Randknoten gegen eine mögliche Verdrehung lagegesichert werden.

\section{3 Überprüfung früherer Reparaturen}

Mit besonderer Sorgfalt sind frühere Erweiterungs- und Reparaturmaßnahmen zu prüfen. Diese können mitunter erhebliche statische Probleme herbeiführen. Wie aufgezeigt wurde, ist die Art der Lagerung der Tonnenschale von entscheidender Bedeutung für deren Standsicherheit: schon kleinere Veränderungen können diese gefährden. Ebenso verhält es sich mit der schalengerechten Lasteinleitung: Dachlasten müssen an den Knotenpunkten des Stabnetzwerks übertragen werden, größere Punktlasten sind $\mathrm{zu}$ vermeiden.

Als Beispiel hierfür sind die Hallen in Oberschleißheim zu nennen. Nach den Kriegsschäden wurde das Dach neu eingedeckt. Dabei wurden Dachlatten etwa in Lamellenmitte, also zwischen den Verbindungsknoten, in Längsrichtung der Halle aufgelegt, um vermutlich die Dachhaut etwas „runder“ zu gestalten (Bild 10). Dies bedeutet aber, dass ca. die Hälfte aller Dachlasten (Eigengewicht, Schnee, Wind) dort eingeleitet wird. Dabei ist nicht nur die Tatsache ungünstig, dass an dieser Stelle kein Knick im Polygonzug des Stabnetzes vorhanden ist - die Belas-

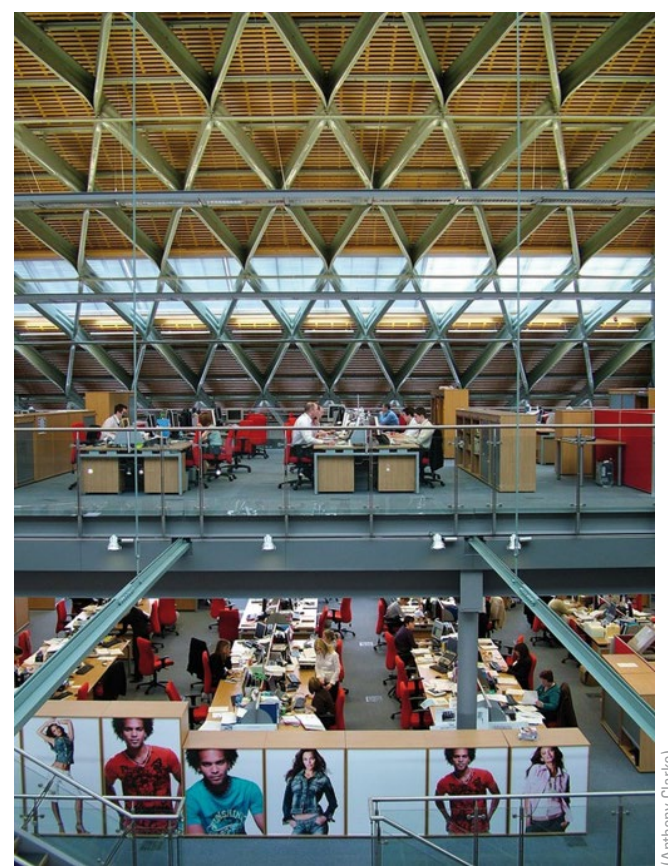

Bild 14 Innenraum des „Skyways House“ in Liverpool, sanierte Lamellendachkonstruktion, Sanierung 2006

Interior of the "Skyways House" in Liverpool, restored lamella roof construction, restoration 2006

tung also anstelle von Drucknormalkräften Biegung hervorruft -, sondern auch, dass die Wirkungslinie der Last deutlich exzentrisch zum Schubmittelpunkt der Lamellen liegt. Der negative Effekt der räumlichen Kippung (Abschn. 2.1) verstärkt die Wirkung der Gravitationslasten je nach Orientierung der Lamelle noch zusätzlich.

\subsection{Prinzipielle Instandsetzungskonzepte}

Zur Sicherstellung einer dauerhaften Tragfähigkeit können grundsätzlich zwei verschiedene Konzepte ggf. auch in Kombination verfolgt werden:

1. Neues, lastübernehmendes Subsidiärtragwerk

2. Austausch/Reparatur geschädigter Bauteile

Es muss für jeden Einzelfall abgewogen werden, mit welcher Lösung den Anforderungen aus Denkmal- und anderen Genehmigungsbehörden sowie der Bauherrn bzw. Nutzer am besten entsprochen werden kann.

Auf dem ehemaligen Junkers-Werksgelände in Dessau ist eine 2012 durchgeführte Instandsetzung durch bereichsweisen Austausch von Lamellen und Unterpfetten zu besichtigen, bei der das Erscheinungsbild der Halle weitgehend unverändert geblieben ist (Bild 13).

Das Skyways House in Liverpool ist eine gelungene Transformation eines ehemaligen Hangars in ein Bürogebäude (Bild 14). Das mehrfach ausgezeichnete Projekt ist nicht nur aus architektonischer Sicht ein gelungenes Beispiel für eine Umnutzung. 


\section{Literatur}

[1] Schmitt, G.: Hugo Junkers - Ein Leben für die Technik. Planegg: Aviatic-Verlag, 1991.

[2] Riess, C.: Die Junkers Tragödie. Münchner Illustrierte (1955), Nr. 25-33.

[3] Tutsch, J.; ToRnack, S.; Barthel, R.: Aus der Luft gegriffen? - Die Lamellenhallen des Luftfahrtpioniers Hugo Junkers. Detail (2015), H. 10, S. 963-970.

[4] Lauff, S.: Der Traum vom Bauen. Berlin: Rhombos-Verlag, 2001.

[5] Patentschrift 508 475, Reichspatentamt, patentiert vom 26.02.1929. http://depatisnet.dpma.de (DE000000508475A).

[6] Villain, J.; Ndogmo, J.: Werkstoffuntersuchung an einer Unterpfette der Junkers-Lamellenhalle in Oberschleißheim. Bericht (unveröffentlicht), 10.04.2016.

[7] Allen, J.: A short history of ,Lamella' roof construction. Transactions of the Newcomen Society 71 (1999-2000), No. 1, pp. 1-29.

[8] ToRnAcK, S.: Der moderne Ingenieurbau der Junkers-Werke in Dessau - Zur Stellung der Metallarchitektur von Hugo Junkers und seiner Werke in der Architekturgeschichte. Masterthesis (unveröffentlicht), HTWK Leipzig, 2012.

[9] Paulssen, O.: Junkers-Lamellendach. Zeitschrift des Vereins Deutscher Ingenieure (1926), Berlin, Bd. 71, Nr. 41, S. 1364-1365.
[10] Bletzinger, K.-U.; Koschnick, F.; Bischoff, M.: Theorie der Tonnenschalen. In: Barthel, R. (Hrsg.), Eladio Dieste Form und Konstruktion, Darmstadt: Verlag Das Beispiel, 2001

[11] Föppl, A.: Das Fachwerk im Raume. Leipzig: Teubner, 1892.

[12] Otzen, R.: Die statische Berechnung der Zollbau-Lamellendächer. Der Industriebau (August-September 1923), H. 12, S. 96-103.

\section{Autoren}

Dipl.-Ing. Joram Tutsch

Andreas Hipper B.Sc.

Dipl.-Ing. Eike Schling

Prof. Dr.-Ing. Rainer Barthel

Technische Universität München

Fakultät für Architektur

Lehrstuhl für Tragwerksplanung

Arcisstraße 21

80333 München

joram.tutsch@tum.de 